\title{
WHO Guidelines on Community-Level Interventions to Manage Declines in Intrinsic Capacity: The Road to Prevention Cognitive Decline in Older Age?
}

\author{
B. Vellas ${ }^{1}$, D. Scrase ${ }^{2}$, G.A. Rosenberg' 3 , S. Andrieu', I. Araujo de Carvalho ${ }^{5}$, L.T. Middleton ${ }^{6}$

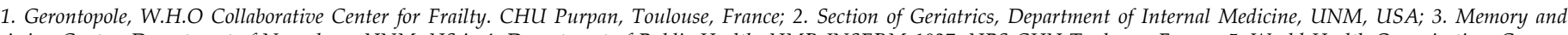

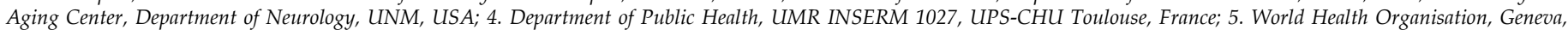 \\ Switzerland; 6. School of Public Health, Imperial College, London, United Kingdom, London, United Kingdom \\ Corresponding Author: B. Vellas, Gerontopole, W.H.O Collaborative Center for Frailty. CHU Purpan, Toulouse, France, E-mail address: vellas.b@chu-toulouse.fr
}

The Concept of decline in "Intrinsic Capacities" (IC) from W.H.O Current Aging program $(1,2)$ prompts us to reposition prevention of cognitive decline and memory disorders with advancing age. In the new conceptual framework, Healthy Ageing is not defined as life free of diseases (disease can occur at any age) but, rather, as «the process of developing and maintaining the functional ability that enables wellbeing in older age» (1). This is a significant departure from the key strategy of current health care systems that are mainly designed to identify and treat acute episodes of (and chronic) illnesses, rather than maintaining individuals' Intrinsic Capacities IC across the life course (1). Hence, there is an urgent need to change the strategic emphasis of health care systems from focusing on acute conditions to promoting integrated care aimed at maintaining functional abilities and well-being as an effective approach to promote healthy ageing.

In its new guidelines, WHO defines «intrinsic capacity» as the composite of the individual's physical and mental capacities, such as mobility, cognition, psychological and sensory, as well as vitality. As healthy ageing is, thus, dependent on an individual's IC but also on the environment and interpersonal interactions, the new emphasis on IC should result in the design of interventions aimed at maintaining functional integrity and well-being (2); the inability to undertake various activities of daily living (ADLs) without the assistance of others can result from (and be a sign of) significant loss of IC. With a life course approach, the WHO model of Healthy Ageing suggests that a period of significant loss of IC in old age is often preceded by some degree of early cognitive decline; hence, pointing to the need of designing new approaches to prevent dementia and Alzheimer's disease.

We, hereby, propose that maintaining cognitive functions in older age will require three approaches: 1 . Increase IC cognitive reserve in mid-life and early aging (45-70 years); 2 . Preserve cognitive functions in late aging $(70$ years + ); 3 . Restore cognitive functions, upon the diagnosis of mild cognitive impairment(MCI), frailty or prodromal late onset dementia, such as Alzheimer's disease (AD).

1. Increase IC cognitive reserves in early aging (4570 years). This first step involves action across the life course, such as higher levels of education, prevention of metabolic and vascular diseases, healthy diet and physical exercise (3-5). The recent decline of the incidence and prevalence of $\mathrm{AD}$ observed in epidemiological studies in western Europe and the USA has been interpreted as being the result of such actions (see review ref 6). In parallel, there is a need to design tools aimed at allowing us to monitor IC reserves across life-course, At the 2017 meeting of the WHO Clinical Consortium on Healthy Ageing, the following recommendations were formulated: Measures of intrinsic capacity should be identified that would be "easy, cheap and relevant to our day-to-day life, as well as culturally relevant" for inclusion in an intrinsic capacity assessment tool. Tools would need to be judged as useful and relevant by older people themselves or the community or primary care workers who may not necessarily be health care professionals. The tests should be easily available not solely for their own sake, but as part of a planned intervention. For such tools to be relevant and meaningful in measuring intrinsic capacity, they should, also, be feasible either by self-management or by trained individuals, without requiring the expertise of a health professional. Interventions should be proactively planned to occur sequentially, if and when individuals and/ or professionals detect a deterioration that exceeds the norm. New tools of measuring IC and cognitive function, using self-administered methodologies, may include new technologies similar to those that are currently available for physical activity and nutrition, such as mobile app applications (7). The European Commission has recently issued a large call towards such methodologies and devices, to address both cognitive functions and mobility.

2. Preserve cognitive functions in late ageing (70 years + ). It is generally acknowledged that the risk of 
dementia doubles every five years, after the age of 65 and that individuals are more prone to some degree of cognitive decline after the age of 75 . We have shown, in the New Mexico Aging Process study (8), that the yearly. incidence of cognitive decline is $14 \%$ over the age of 78 years, compared to $7 \%$ for those younger than 78 years old. Furthermore, only $24 \%$ of the older old ( $>78$ years) were able to regain cognitive and functional abilities, as compared to $64 \%$ of those below 78 years. Moreover, the older old subjects were more likely to have both cognitive decline and motor manifestations, such as slow gait speed, than younger individuals. These findings point to the need of developing tools to closely monitor cognitive and memory functions, in older adults, using self-management strategies or with the help of health professional when needed. Of the myriad of available cognitive batteries, easy-to-use tests that would allow assessments through mobile apps or tablets in a reliable manner should be used and validated for optimal sensitivity and specificity.

3. Restore cognitive functions, once MCI or «prodromal» late onset Dementia, (such as AD) occurs. Ideally, we should be able to restore cognitive functions, based on the "precision medicine» model, i.e. being able to decide on the right treatment for the right patient. Such an approach, already in best medical practice for several cancer forms, would pre-require a diagnosis of the precise disease profile (or sub-type) based on the individual's biomarker based -signature; this would lead to the choice of the right treatment, targeting the specific biological mechanisms underlying disease etiology for the optimal «right therapy». However, the reality of our understanding of the nosology of $\mathrm{AD}$ and other late onset dementias still remains partial and incomplete. The main nosological classification includes AD, seen as a disease related to abnormal cerebral amyloid and -tau load, vascular dementia, Lewy Body dementia, or decline due to the so-called «Cognitive Frailty Syndrome» (9). Unlike the early onset hereditary AD form, the late onset and commonest $\mathrm{AD}$ is thought to be complex; like many other common disorders, such as Hypertension and type II diabetes, resulting from complex interactions (over time) of multiple genetic and acquired, (including environmental) factors, with limited understanding of the precise nature of these factors, their respective effect(s) and the choreography and/or temporal pattern of key biological events. Furthermore, there is still a significant gap in our understanding on how biological and pathological findings translate into memory, cognitive, behavioral and functional abnormalities.

Promoting Integrated Care to Maintain Cognitive functions with Advancing Age versus Early diagnosis of Alzheimer's Disease? There are, still, several barriers for the early diagnosis of Alzheimer's disease, notwithstanding the important limitations of our understanding of disease nosology. The first barrier is the complexity (and poor specificity) of the terminology used, whilst concepts are continuously evolving. The use of terms such as «pre-clinical Alzheimer's disease stages» age-related decline, MCI (early and late), MCI due to $\mathrm{AD}$, prodromal $\mathrm{AD}$ and others are confusing and may explain why primary care and other physicians are often reluctant to make a diagnosis of early cognitive decline. A second barrier is the lack of proven disease modifying therapies for $\mathrm{AD}$ and other late onset dementias. Following a series of failures of randomized control trials (RCT) in patients with AD, over the last 25 years, there is still hope for positive signals in current RCTs in pre-clinical at risk individuals. Thirdly, the currently available AD biomarkers, such Positron Emission Tomography (PET) or cerebrospinal fluid (CSF) profiling for amyloid or tau are not widely available or accessible, they are expensive and not devoid of risks. In this context, a defined set of actions centered to monitor and maintain function throughout the life span and advancing age, could be a first important step. A successful toolkit would need to be comprehensive, focusing not only on cognition but also on the other IC functions, such as mobility, psychological and mood status, vitality, hearing and vision. All these IC functions can also have detrimental effects on memory and cognition and should be part of an integrated care model. The dream is that, sooner rather than later, new scientific discoveries will shed light into the disease complexity and pave the way for evidence-based preventive strategies and more effective precision- medicine therapies for dementia sufferers; the right medicine for the right patient.

\section{References}

1. WHO Clinical Consortium on Healthy Ageing 2017 - report of consortium meeting 21 and 22 November 2017 in Geneva, Switzerland. Geneva: World Health Organization; 2018. Licence: CC BY-NC-SA 3.0 IGO.

2. Cesari M1,2,3,4, Araujo de Carvalho I5, Amuthavalli Thiyagarajan J5, Cooper C6, Martin FC7, Reginster JY8, Vellas B1,2, Beard JR5. Evidence for The Domains Supporting The Construct of Intrinsic Capacity. J Gerontol A Biol Sci Med Sci. 2018 Feb 2. doi: 10.1093/gerona/gly011. [Epub ahead of print]

3. Ngandu T, Lehtisalo J, Solomon A, Levälahti E, Ahtiluoto S, Antikainen R, Bäckman L, Hänninen T, Jula A, Laatikainen T, Lindström J, Mangialasche F, Paajanen T, Pajala S, Peltonen M, Rauramaa R, Stigsdotter-Neely A Strandberg T, Tuomilehto J, Soininen H, Kivipelto M. A 2 year multidomain intervention of diet, exercise, cognitive training, and vascular risk monitoring versus control to prevent cognitive decline in at-risk elderly people (FINGER): a randomised controlled trial. Lancet. 2015 Jun 6;385(9984):225563. doi:10.1016/S0140-6736(15)60461-5. Epub 2015 Mar 12. PubMed PMID: 25771249.

4. Andrieu S(1), Guyonnet S(2), Coley N(3), Cantet C(2), Bonnefoy M(4), Bordes $\mathrm{S}(5)$, Bories L(6), Cufi MN(7), Dantoine T(8), Dartigues JF(9), Desclaux F(10), Gabelle A(11), Gasnier Y(5), Pesce A(12), Sudres K(13), Touchon J(14), Robert $\mathrm{P}(15)$, Rouaud $\mathrm{O}(16)$, Legrand $\mathrm{P}(17)$, Payoux $\mathrm{P}(18)$, Caubere JP(19), Weiner M(20), CarriéI, Ousset PJ(2), Vellas B(2); MAPT Study Group. Effect of longterm omega 3 polyunsaturated fatty acid supplementation with orwithout multidomain intervention on cognitive function in elderly adults withmemory complaints (MAPT): a randomised, placebo-controlled trial. Lancet Neurol. 2017 May;16(5):377-389. doi: 10.1016/S1474-4422(17)30040-6. Epub 2017 Mar 27.

5. Barbera M, Mangialasche F, Jongstra S, Guillemont J, Ngandu T, Beishuizen C, Coley N, Brayne C, Andrieu S, Richard E, Soininen H, Kivipelto M; HATICE study group. Designing an Internet-Based Multidomain Intervention for the Prevention of Cardiovascular Disease and Cognitive Impairment in Older Adults: The HATICE Trial. J Alzheimers Dis. 2018;62(2):649-663. doi: 10.3233/ JAD-170858. PubMed PMID: 29480185. 
6. Winblad B, Amouyel P, Andrieu S, Ballard C, Brayne C, Brodaty H, CedazoMinguez A, Dubois B, Edvardsson D, Feldman H, Fratiglioni L, Frisoni GB, Gauthier S, Georges J, Graff C, Iqbal K, Jessen F, Johansson G, Jönsson L, Kivipelto M, Knapp M, Mangialasche F, Melis R, Nordberg A, Rikkert MO, Qiu C, Sakmar TP, Scheltens P, Schneider LS, Sperling R, Tjernberg LO, Waldemar G, Wimo A, Zetterberg H. Defeating Alzheimer's disease and other dementias: a priority for European science and society. Lancet Neurol. 2016 Apr;15(5):455-532. doi: 10.1016/ S1474-4422(16)00062-4. Review.

7. Michel J.-P. Mobile application removes societal barriers to P4 medicine. J Frailty Aging. 2017;6(4):216-218. doi: 10.14283/jfa.2017.43.

8. Qualls C, Waters DL, Vellas B, Villareal DT, Garry PJ, Gallini A, Andrieu
S. Reversible States of Physical and/or Cognitive Dysfunction: A 9-Year Longitudinal Study. J Nutr Health Aging. 2017;21(3):271-275. doi: 10.1007/ s12603-017-0878-3 PubMed PMID: 28244566.NMAPS

9. Donohue MC, sperling RA, Salmon DP, Rentz DM, Raman R, Thomas RG, Weiner M,Aisen PS. Australian Imaging, Biomarkers, and Lifestyle Flagship Study of Ageing;Alzheimer's Disease Neuroimaging Initiative; Alzheimer's Disease Cooperative Study. The preclinical Alzheimer cognitive composite: measuring amyloid-related decline. JAMA Neurol. 2014 Aug;71(8):961-70. doi: 10.1001/jamaneurol.2014.803. PubMed PMID: 24886908; PubMed Central PMCID: PMC4439182. 